\title{
Low-Resolution STELab IPS 3D Reconstructions of the Whole Heliosphere Interval and Comparison with in-Ecliptic Solar Wind Measurements from STEREO and Wind Instrumentation
}

\author{
M.M. Bisi • B.V. Jackson • A. Buffington · J.M. Clover • \\ P.P. Hick $\cdot$ M. Tokumaru
}

Received: 4 December 2008 / Accepted: 31 March 2009 / Published online: 11 April 2009

(c) The Author(s) 2009. This article is published with open access at Springerlink.com

\begin{abstract}
We present initial 3D tomographic reconstructions of the inner heliosphere during the Whole Heliosphere Interval (WHI) - Carrington Rotation 2068 (CR2068) - using Solar-Terrestrial Environment Laboratory (STELab) Interplanetary Scintillation (IPS) observations. Such observations have been used for over a decade to visualise and investigate the structure of the solar wind and to study in detail its various features. These features include co-rotating structures as well as transient structures moving out from the Sun. We present global reconstructions of the structure of the inner heliosphere during this time, and compare density and radial velocity with multi-point in situ spacecraft measurements in the ecliptic; namely STEREO and Wind data, as the interplanetary medium passes over the spacecraft locations.
\end{abstract}

STEREO Science Results at Solar Minimum

Guest Editors: Eric R. Christian, Michael L. Kaiser, Therese A. Kucera, O.C. St. Cyr

M.M. Bisi $(\bowtie) \cdot$ B.V. Jackson · A. Buffington · J.M. Clover · P.P. Hick

Center for Astrophysics and Space Sciences, University of California, San Diego,

9500 Gilman Drive \#0424, La Jolla, CA 92093-0424, USA

e-mail: mmbisi@ucsd.edu

M.M. Bisi

e-mail: Mario.Bisi@gmail.com

B.V. Jackson

e-mail: bvjackson@ucsd.edu

A. Buffington

e-mail: abuffington@ucsd.edu

J.M. Clover

e-mail: jclover@ucsd.edu

P.P. Hick

e-mail: pphick@ucsd.edu

M. Tokumaru

Solar-Terrestrial Environment Laboratory, Nagoya University, Furo-cho, Chikusa-ku, Nagoya

464-8601, Japan

e-mail: tokumaru@stelab.nagoya-u.ac.jp 
Keywords Solar wind - Inner heliosphere - IPS · STELab - STEREO · PLASTIC · Wind · SWE $\cdot$ WHI

\section{Introduction}

Interplanetary scintillation (IPS) has been used for inner-heliospheric observations of the solar wind since its discovery in the 1960s (e.g. Hewish, Scott, and Wills, 1964; Coles and Rickett, 1976; Kojima and Kakinuma, 1987; Manoharan and Ananthakrishnan, 1990; Breen et al., 2002; Jones et al., 2007). IPS arises from scattering by small-scale $(\sim 150 \mathrm{~km})$ density inhomogeneities in the solar wind flowing approximately radially outward from the Sun and is seen as the rapid variation of a radio signal received at Earth from a distant compact naturally-occurring radio source. IPS observations allow the solar wind speed to be inferred over a wide range of heliocentric distances and also over all heliographic latitudes dependent upon source strength and observing frequency (e.g. Hewish, Scott, and Wills, 1964; Coles and Maagoe, 1972; Kojima et al., 2004; Bisi et al., 2007b). Using the scintillation level, converted to $g$-level as a proxy for density (see Equation (1) later), an underlying solar wind density can also be deduced over the same range from IPS observations (e.g. Jackson et al., 1998 and references therein; Hick and Jackson, 2004; Jackson and Hick, 2005).

When multiple radio antennas/telescopes are used simultaneously to observe the same source in the sky, and the separation of the raypaths from antenna to source in the plane of the sky lies approximately along the radial direction from the Sun (i.e. along the assumed solar wind flow direction), a high degree of correlation may be observed between the scintillation patterns in the signal recorded at each of the observing sites (e.g. Armstrong and Coles, 1972). The time lag for which maximum cross-correlation occurs (taking into account 'plane-of-sky' assumptions) then provides an estimate of the outflow speed of the irregularities producing the scintillation (e.g. Breen et al., 2006; Bisi et al., 2006). More sophisticated methods fitting the observed auto- and cross-correlation spectra with the results from a weak-scattering model have also been adopted for IPS data analyses (e.g. Coles, 1996; Klinglesmith, 1997; Bisi et al., 2007a; Fallows, Breen, and Dorrian, 2008). In addition, as is done in this paper, the whole heliosphere can be reconstructed in three dimensions from IPS data using a tomographic model which combines many lines of sight to undo the line-ofsight (LOS) integration (see later). Figure 1 gives a schematic example of IPS signals being received using two radio antennas (taken from Bisi et al., 2009a; adapted from Bisi et al., 2004 in a similar form). This shows similar amplitude variations with a time lag as they pass across the sky from one antenna's LOS to the other; they are later used to calculate an estimate of the outflow speed (radial velocity). IPS is most sensitive at the raypath's point of closest approach to the Sun (P-Point), and to material flowing perpendicular (or close-to perpendicular) across the LOS.

The primary data discussed in this paper are observations made using the radio arrays of the Solar-Terrestrial Environment Laboratory (STELab) (Kojima and Kakinuma, 1987), University of Nagoya, Japan; the Solar TErrestrial Relations Observatory (STEREO) spacecraft pair (Kaiser, 2005; Kaiser et al., 2008), and the Wind spacecraft (Ogilvie and Desch, 1997). For these latter, we have used data from the Plasma and Suprathermal Ion Composition (PLASTIC) instrumentation (Galvin et al., 2008) aboard the STEREO spacecraft pair, and the Solar Wind Experiment (SWE) (Ogilvie et al., 1995) aboard the Wind spacecraft. Section 2 briefly discusses the conversion to $g$-level from the scintillation level and the threedimensional (3D) tomographic reconstruction method used in this paper. Section 3 describes the Whole Heliosphere Interval (WHI) observations in general. Section 4 describes the in situ measurements and the present comparison. We summarise and conclude in Section 5. 


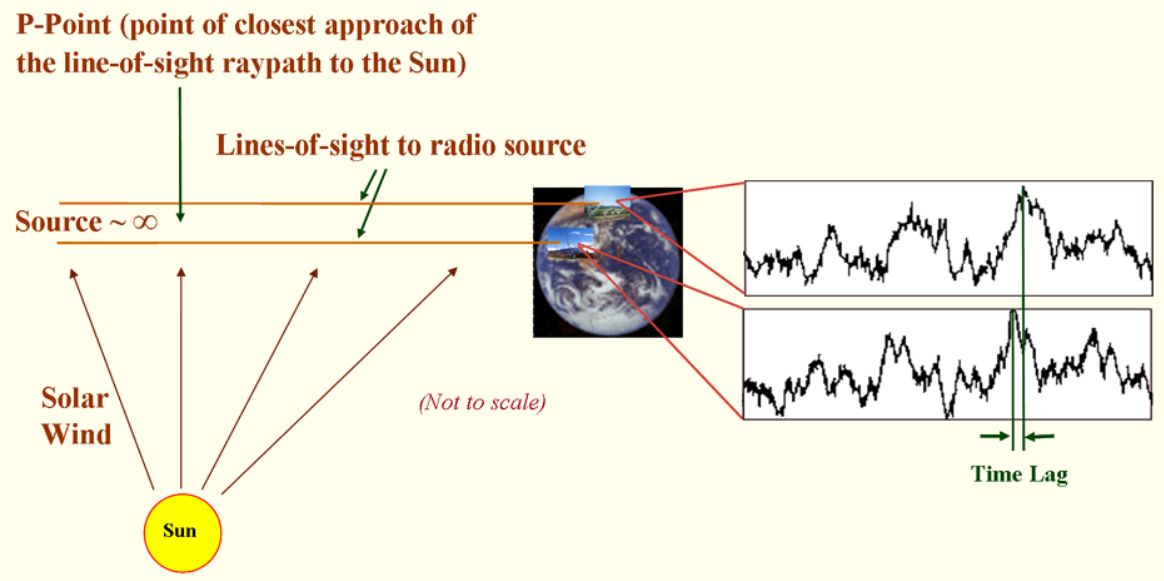

Figure 1 Figure showing the basic principles of multi-site (in this case two) interplanetary scintillation (IPS) through the simultaneous observation of a single radio source (taken from Bisi et al. (2009a); adapted from Bisi et al. (2004), in a similar form). The signal received from a distant, compact source has a variation in amplitude which is directly related to turbulence in the material crossing the LOS, and can thus be related to variations in density. Figure outline originally courtesy of R.A. Fallows (Aberystwyth University).

\section{Calculations and Methods Employed in this Paper}

The 3D velocity reconstructions are based directly on the IPS velocity observations; for 3D density reconstructions the $g$-level provides a proxy for density. This requires a "conversion" from IPS scintillation level, expressed as $g$-level, to density. The resulting reconstructions are of an inner-heliosphere region typically ranging from 15 solar radii $\left(R_{\odot}\right)$ out to approximately 3 astronomical units (AU).

\subsection{Calculation of $g$-Level and Proxy for Density}

Scintillation-level measurements have been obtained using the STELab IPS-dedicated radio antenna at Kiso from 1997 to the present, and more recently (from mid-2002) from the Fuji antenna (see Jackson and Hick, 2005; Bisi et al., 2009b, and references therein). The new Toyokawa site when fully operational should extend the STELab system to observe many more radio sources per day. Another STELab antenna is located at Sugadaira. The original four STELab antennas are pictured in Figure 2. STELab currently typically observes 20 to more than 40 astronomical radio sources per day.

The disturbance factor, or $g$-level, $g$, is defined by Equation (1):

$$
g=m /\langle m\rangle \text {. }
$$

Here, $m$ is the observed scintillation level, and $\langle m\rangle$ is the mean level of scintillation for the source at the elongation at the time of observation. Scintillation-level measurements from STELab are available for each astronomical radio source as an intensity variation of signal strength (resulting from small-scale variations in density, $\Delta N_{\mathrm{e}}$ ). These data are automatically edited to remove any obvious interference. 


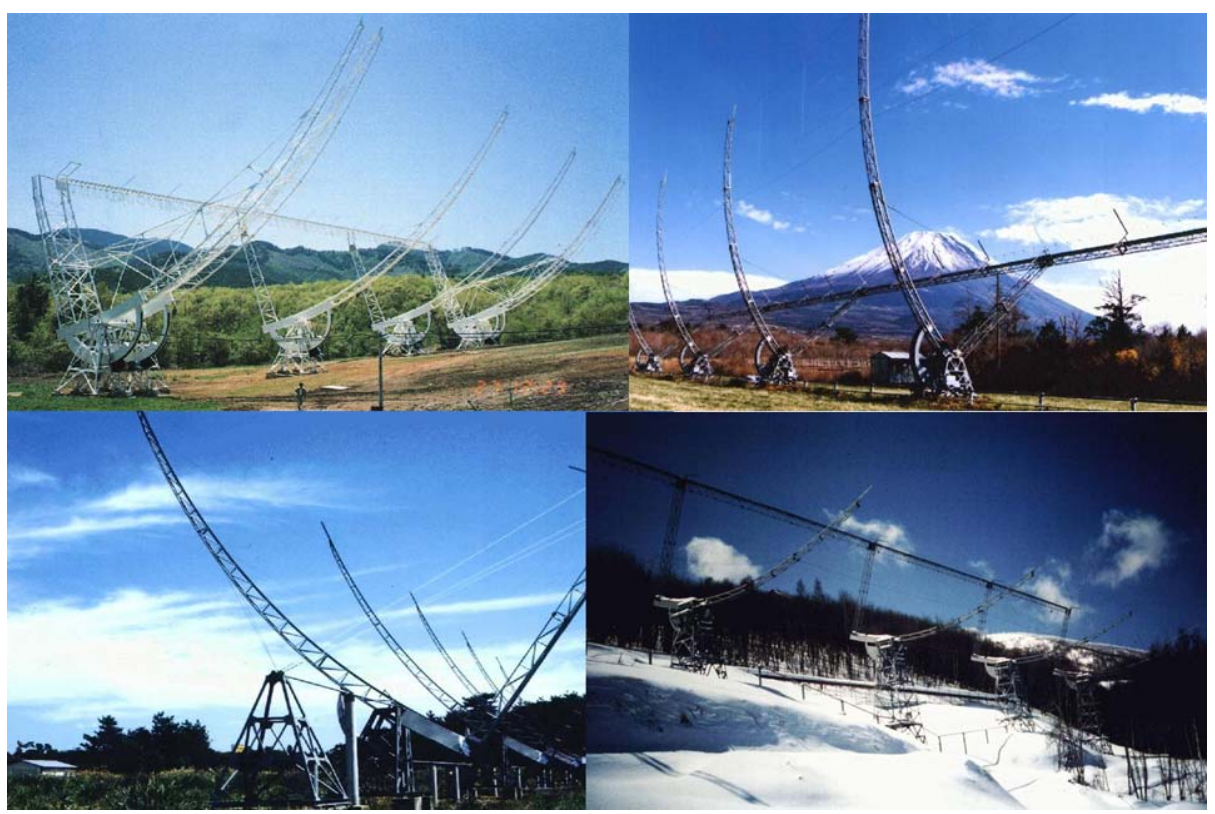

Figure 2 The STELab antennas: Kiso (top left), Fuji (top right), Toyokawa - old antenna (bottom left), and Sugadaira (bottom right) (see http://stesun5.stelab.nagoya-u.ac.jp/uhf_ant-e.html).

Since density values along the LOS are not known a priori, the $g$-level proxy for density is related to small-scale density variations by Equation (2). The small-scale density variations are assumed to have a power law dependence on heliospheric density and heliocentric distance;

$$
\Delta N_{\mathrm{e}}=A_{\mathrm{c}} R^{\mathrm{PWR}} N_{\mathrm{e}}^{\mathrm{PWN}} .
$$

Here, $A_{\mathrm{c}}$ is a proportionality constant, $R$ is the radial distance from the Sun, PWR is the power of the radial falloff, and PWN is the power of the density. $A_{\mathrm{c}}, \mathrm{PWR}$, and PWN are determined using best-fit comparisons with in situ data at $1 \mathrm{AU}$.

Further discussion of determining $g$-level and density can be found in Jackson et al. $(1998,2003$, and references therein). In addition, this and the real-time calculation used for space-weather forecasting at http://ips.ucsd.edu/ can be found in Hick and Jackson (2004, and references therein).

\subsection{The 3D Reconstruction Method}

The 3D tomographic reconstruction uses perspective views of solar co-rotating plasma (Jackson et al., 1998) and of outward-flowing solar wind (Jackson and Hick, 2005) crossing the IPS observing lines of sight from Earth to the radio source. Both radial velocity and density are obtained through the use of a kinematic solar wind model. IPS data are then fitted iteratively to refine this model over 18 iterations to be sure of convergence to a final best-fit solution.

We then compare the resulting reconstructions with hourly-averaged in situ measurements from both the Wind/SWE and STEREO/PLASTIC instruments. Previous comparisons have been with data from the Advanced Composition Explorer - Solar Wind Electron, 
Proton and Alpha Monitor (ACE/SWEPAM) (Stone et al., 1998; McComas et al., 1998); the Ulysses spacecraft (Wenzel et al., 1992); and finally with "ram" pressure measurements inferred from the Mars Global Surveyor magnetometer (Crider et al., 2003) in orbit around Mars with IPS observations made between 1999 and 2004 (Jackson et al., 2007). Jackson and Hick (2005) describe the time-dependent 3D tomographic technique used here (first introduced in 2000).

The 3D reconstructions for the STELab data used here have latitude and longitude digital resolution of $20^{\circ} \times 20^{\circ}$. The one-day time cadence has 6-hour interpolated increments, to yield output four times a day for the modelled structure of the inner heliosphere.

The accuracy of this model is now well confirmed both in the low-resolution form used in this paper and in higher-resolution forms such as for IPS data from the Ootacamund (Ooty) Radio Telescope (ORT) (e.g. Bisi et al., 2009b) or white-light observations from the Solar Mass Ejection Imager (SMEI) (e.g. Jackson et al., 2006, 2008; Bisi et al., 2008). The output of these tomographic reconstructions have successfully provided a "source surface" input into the ENLIL 3D magnetohydrodynamic (MHD) numerical model (e.g. Odstrcil and Pizzo, 2002) which when propagated out through the interplanetary medium, also compares well with in situ measurements (e.g. Bisi et al., 2008).

\section{Observations}

WHI covers Carrington rotation 2068 (CR2068) and runs through the period 20 March 2008 to 16 April 2008 at the Earth (which approximately includes the Wind spacecraft orbiting the Sun-Earth $L_{1}$ point). Since Carrington longitude is measured as viewed from Earth for each rotation, the dates for CR2068 for spacecraft outside of the Sun-Earth line will differ since the $0^{\circ}$ longitude line will pass under them at different times. For STEREO-A (ahead) the dates are 21 March 2008 to 18 April 2008, and for STEREO-B (behind) they are 18 March 2008 to 14 April 2008. Due to the 2008 STELab observing season running from April/May through November/December each year, the antennas were not operational until 3 April 2008 (approximately half-way through the WHI time interval), these then unfortunately suffered severe mechanical failures shortly after the WHI time period on 22 April 2008. Figure 3 shows the directly-observed synoptic Sun coverage of velocity using IPS, as determined by projecting a Gaussian-weighted circle of $10^{\circ}$ from the LOS P-Point to the source-surface map using timing from that point to the surface corresponding to the IPS velocity observation. Coverage is incomplete. The number of velocity observations exceeds that of scintillation-level ( $g$-level) observations due to hardware difficulties at STELab. The bulk of the observations used to reconstruct the latter half of CR2068 (WHI) - as discussed in this paper - came from lines of sight that predominantly map back to the later portion of this Carrington rotation since there were no IPS observations which took place during CR2067 and very few during CR2069. Figure 4 shows plots of the lines of sight projected to the source surface for all the data used in the 3D reconstructions, both $g$-level (top) and velocity (bottom) sources (see Jackson and Hick, 2005 for another example). As can be seen in the figure, the velocity coverage is far greater over the two Carrington rotations used for the tomography (CR2067.50 to CR2069.50) than that of $g$-level coverage, particularly at the beginning of the time interval. The lack of projected lines of sight in early CR2068 results in data gaps. This is particularly noticeable to the west of the Sun-Earth line. In addition, there is little coverage in either CR2067 or CR2069. These plots show the greater number of velocity observations used in the 3D reconstructions as compared with those of $g$-level. Figure 5 shows the weighted sum of LOS crossings after projection onto the source surface 


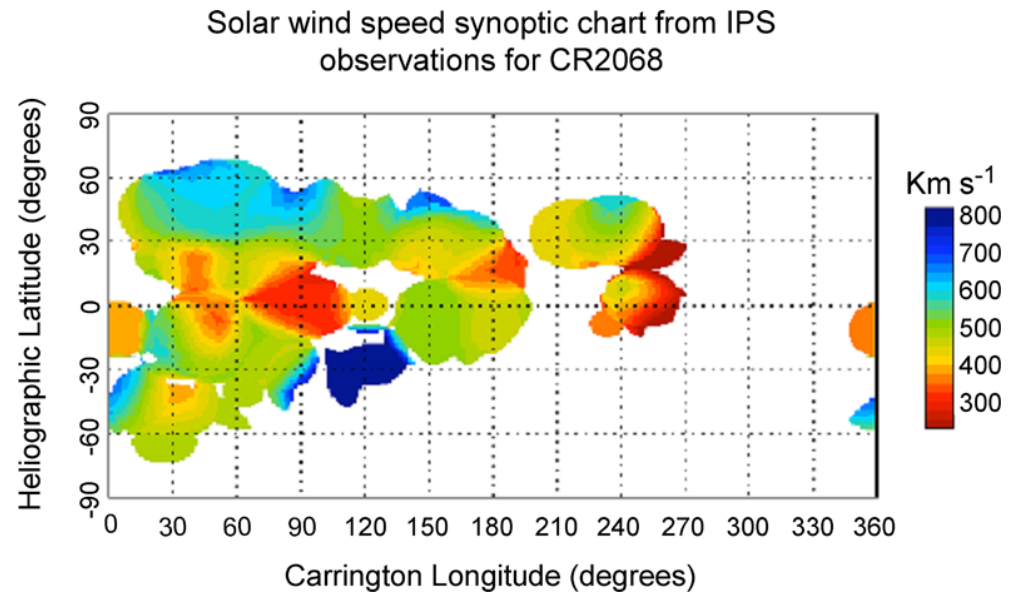

Figure 3 The direct IPS synoptic Sun coverage for resulting velocity structure as made by the STELab IPS arrays during WHI. Velocity for each position is assumed to remain constant and radial. The velocity observations are mapped back to the source surface using the P-Point approximation as the location along the LOS. A $10^{\circ}$ spread function (in the form of a Gaussian) is then used for both longitude and latitude to blend neighbouring IPS observations onto the Carrington source-surface map.

of all the IPS data used for this reconstruction. The greater the number of weighted LOS crossings, the higher the confidence in the reconstructions; thus, these plots provide confidence levels for the reconstruction in both $g$-level (top) and velocity (bottom) projected onto the source surface. These plots again show that more information contributes to reconstruct the velocity than the density, especially to the west of the Sun-Earth line at the beginning of this time interval. However, from the observations which did take place, the inner heliosphere was successfully reconstructed (at least near the ecliptic plane) at the low digital spatial resolutions and temporal cadence using the time-dependent 3D tomography (Figures 6 and 7). Here, the density and velocity both have the same 3D-reconstructed resolutions.

Two main features can be observed; the first appears to be a co-rotating interaction region (CIR), and the second a coronal mass ejection (CME). These respectively can be seen in ecliptic-cut extractions from the 3D tomography in both velocity and density in Figures 6 and 7. These figures cover a period of three or four days centred on 03:00 UT for each day. In all cases, the view covers the ecliptic plane viewed from the north out to a distance of 1.5 AU from the Sun. Gaps and white areas in the ecliptic cuts are places where there were insufficient data to fully reconstruct the inner heliosphere. There is also an inner-exclusion zone outside of which the 3D reconstructions form. STELab observations take place at a frequency of $327 \mathrm{MHz}$ and extend outward from $11.5^{\circ}$ elongation (or approximately from $45 R_{\odot}$ for P-Point distance from the Sun).

Figure 6 shows a CIR-like structure over a period of three days from 4 April 2008 to 6 April 2008. There is higher density material with slow-to-intermediate velocities out at the front of the co-rotating feature, followed by lower-density higher-speed material flowing out behind. The high-speed stream can also be seen to be rotating (anti-clockwise) throughout the three days shown here. On the first day, 4 April 2008, both the Earth (and hence the Wind spacecraft orbiting the Sun-Earth $L_{1}$ point) and the STEREO-A spacecraft are immersed 

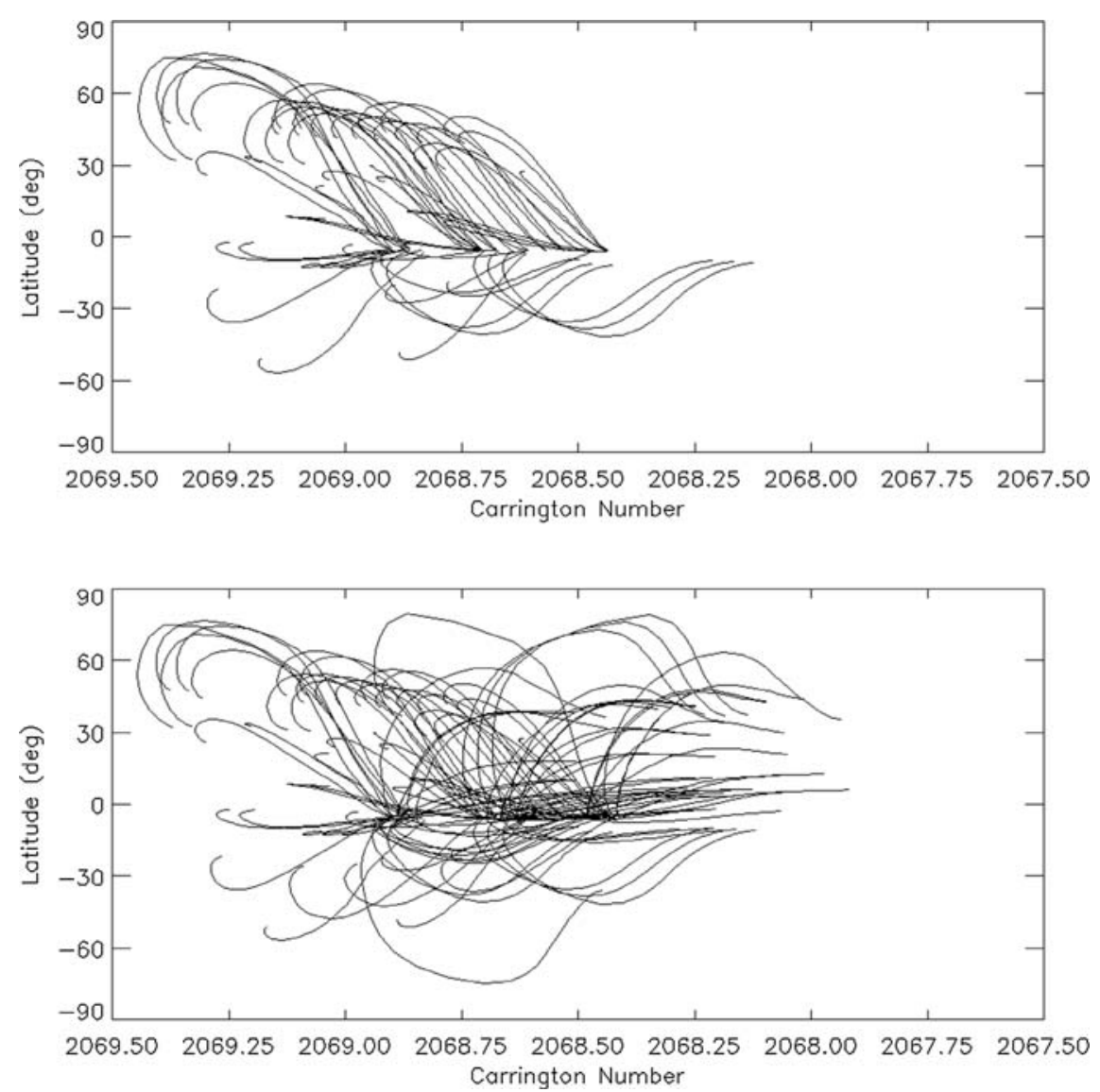

Figure 4 The STELab IPS lines of sight used by the 3D tomography from scintillation level (top) and velocity (bottom) observations mapped back to $15 R_{\odot}$ which is the source surface (lower boundary) in the $3 \mathrm{D}$ tomography. As can be seen, the velocity coverage is far greater over the two Carrington rotations used for the tomography (CR2067.50 to CR2069.50) than that of $g$-level coverage; thus resulting in some gaps in the density $3 \mathrm{D}$ reconstructions as seen in Figure 6.

in the higher-density material and slower wind speed, while the STEREO-B spacecraft is starting to be immersed in higher-speed flow but at a lowered density. By 24-hours later on 5 April 2008, an even lower-density region is approaching the STEREO-B spacecraft with an increased flow speed now seen at all three locations increasing in its magnitude from the STEREO-A spacecraft to Earth, and again from Earth to the STEREO-B spacecraft; the slower stream has now rotated around to the west of the Sun-Earth line a little more. There was also a weak drop in the Dst index (disturbance storm time index) seen on 5 April 2008 signifying a weak geo-effective storm created by the CIR. By the final day shown here, 6 April 2008, all three locations have a lowered density while the higher-speed flow is still rotating around and beginning to engulf the Earth as well as the STEREO-B spacecraft.

At this time, high-speed streams were originating in equatorial coronal holes. These could be seen faintly in coronagraph observations and Sun-surface synoptic maps which is where the CIR-type feature may have originated. 
g LOS crossings at $0.07 \mathrm{AU}$

80

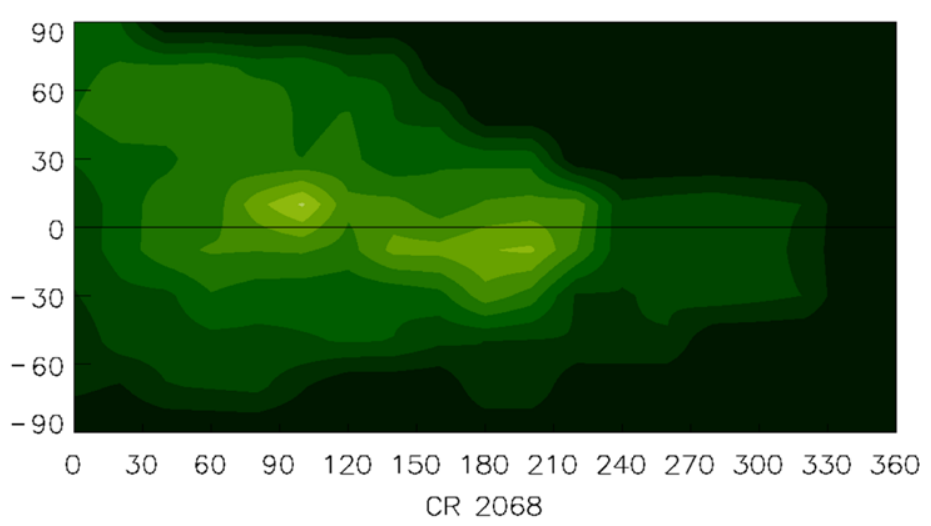

$\checkmark$ LOS crossings at $0.07 \mathrm{AU}$

80

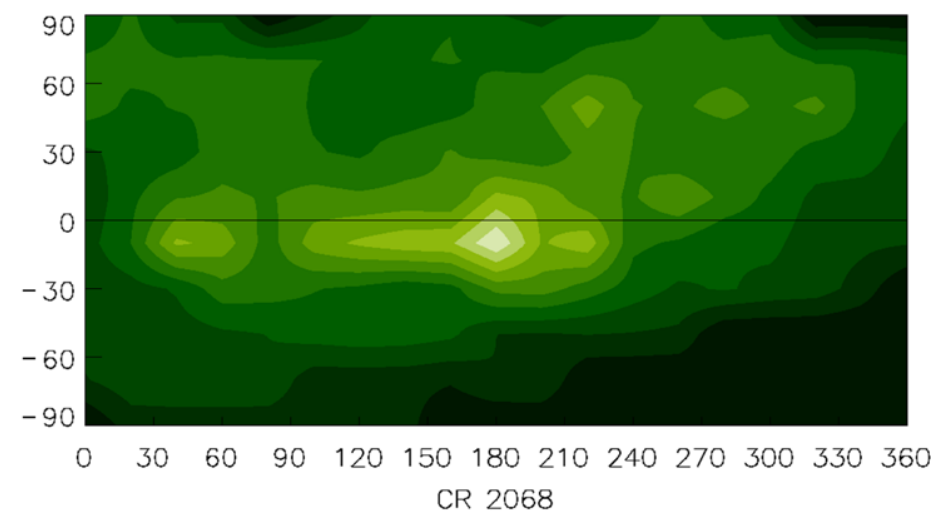

Figure 5 The summed STELab IPS LOS crossings from $g$-level (top) and velocity (bottom) observations (in each $20^{\circ} \times 20^{\circ}$ reconstructed bin) mapped back to the source surface. Those portions of these maps having the largest number of LOS crossings have the highest confidence level. As is seen here, when compared with the $g$-level plot, the velocity confidence is greater near the sub-Earth latitude (the ecliptic) and more extensive to the west (larger longitudes, in the earlier parts of CR2068). The source-surface LOS crossings for each time-reconstructed Carrington map are normalised per day crossings and for the Gaussian temporal and spatial filters. Approximately 20 days of observations are used to make the Carrington plots shown here. In the tomography at STELab resolutions, the standard deviations of the Gaussian filters are respectively 0.65 day and $14^{\circ}$; see Jackson et al. (2003) for further details.

Figure 7 shows a transient feature, possibly two; one with increased density already extended beyond $1 \mathrm{AU}$ west of the Sun - Earth line (which could also be part of a co-rotating region), and another density increase not yet at $1 \mathrm{AU}$ heading roughly along the Sun - Earth 
line and finally merging with the first feature out to the west. Several weak CMEs observed by the Large Angle Spectroscopic COronagraph (LASCO) occurred between 7 April 2008 and 11 April 2008 at position angles allowing for the first enhanced density above, but no halo-type CME that could account for the second. A west-limb CME that was seen by SOlar and Heliospheric Observatory (SOHO), Hinode, and STEREO from active region (AR) 10989 on 9 April 2008 at 10:26:05 UT (first appearance in LASCO C2 - taken from the SOHO/LASCO CDAW CME catalogue at http://cdaw.gsfc.nasa.gov/CME_list/) is the likely candidate for these west-limb interplanetary reconstructions seen in the ecliptic cuts between 12 April 2008 and 13 April 2008. Figure 7 shows ecliptic cuts for 12 April 2008, 13 April 2008, 14 April 2008, and 15 April 2008 all centred at 03:00 UT as in Figure 6. Higher-resolution tomography with data from other IPS sources or SMEI could likely better distinguish where these transient features originated.

Velocity structure is somewhat more complex than density structure for these solarminimum conditions. Regions of increased and decreased velocities inside of $1 \mathrm{AU}$ bear some correlation with weak events seen in LASCO; also some equatorial extensions of coronal holes still exist throughout this Carrington rotation.

\section{Comparison with In Situ Measurements}

The 3D reconstructions permit extraction of a time series for both velocity and density values at any given location within the reconstructed volume. Figures 8, 9, and 10 are summaries of the portion of CR2068 for which there were STELab observations; these show reconstruction comparisons with in situ measurements from Wind and STEREO (A and B) spacecraft respectively for both parameters. The in situ data have been re-averaged to a oneday cadence commensurate with that of the 3D STELab tomographic reconstruction digital cadence used here.

For a co-rotating structure, since STEREO-B lags behind STEREO-A in the Sun's rotational direction, features sweep over STEREO-B first, then pass the Wind spacecraft and the Earth, and finally pass STEREO-A. For a transient structure, the order in which features arrive at various spacecraft or the Earth depends on the orientation and launch direction of the particular transient.

\subsection{Comparison with Wind/SWE In Situ Measurements}

A preliminary comparison with ACE Level-0 data and Wind in situ measurements is given by Bisi et al. (2009b). In this paper, looking at only the Wind/SWE data, we see that both the velocity and density in situ measurements compare well with the 3D tomography-extracted values and result in good correlations for both parameters.

An earlier peak in density around 4 April 2008 is reproduced well by the tomography. A weaker transient-associated peak later around 13 April 2008 is shown in the tomography that is observed as a small in situ density enhancement in Wind/SWE data.

\subsection{Comparison with STEREO/PLASTIC}

Figures 9 and 10 respectively compare in situ measurements with time-series extractions from the 3D tomography for STEREO-A and STEREO-B. In Figures 9 and 10, velocity measurements at each spacecraft are similar to those extracted from the tomography and give very good correlation for both STEREO-A and STEREO-B, respectively. Again, the correlation is good for STEREO-B density comparison, but the density measured by STEREO-A 


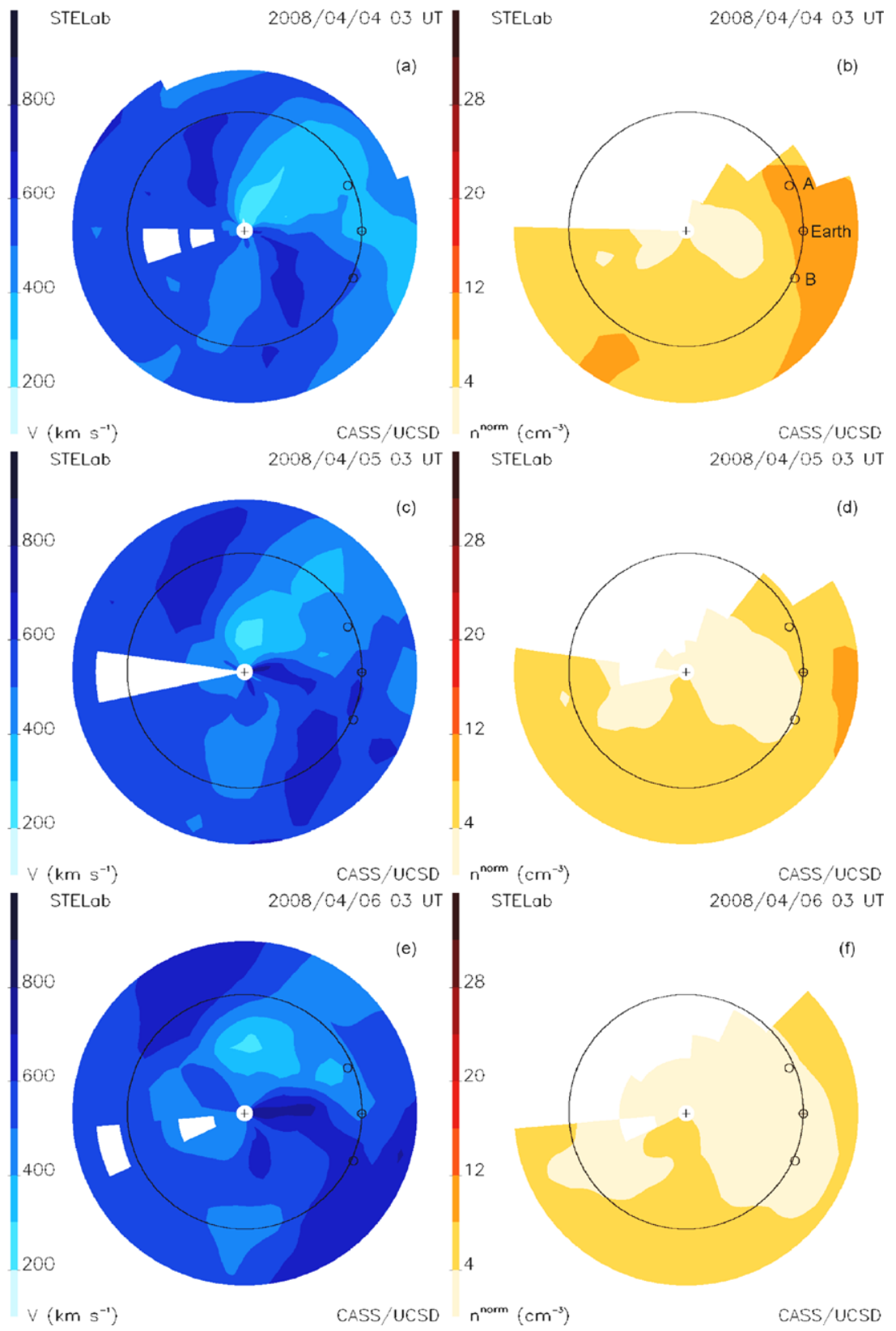

Figure 6 Figure displaying ecliptic cuts through the 3D tomography looking down from north of the ecliptic in both velocity (a), (c), and (e), and density (b), (d), and (f). A period of three days is shown here centred on 03:00 UT for each of the days and the days are marked from 4 April 2008 through 6 April 2008. The Sun is at the centre marked with a + and an ellipse maps out the Earth's orbital path in the ecliptic, the $\oplus$ as the Earth, and two small circles representing the two STEREO spacecraft (which are labelled accordingly in the top-right image); one out ahead of the Earth (STEREO-A) and one lagging behind the Earth (STEREO-B). The Earth's orbital direction here is in the anti-clockwise direction. White space signifies gaps in the 3D reconstruction due to insufficient data points to successfully complete the inner heliosphere in the 3D volume. Since the numbers of velocity observations are more numerous during this time, the density ecliptic cuts have more gaps to the west of the Sun-Earth line, i.e. in the vicinity of the STEREO-A spacecraft. 

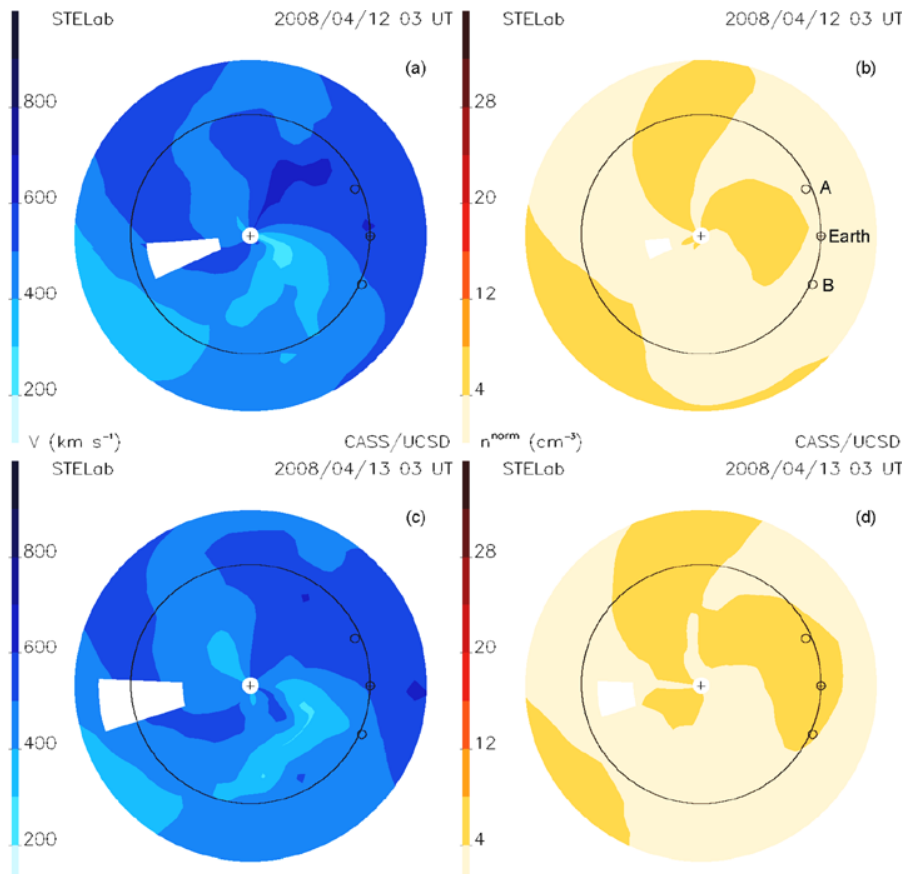

CASS/UCSD $2008 / 04 / 1303$ UT
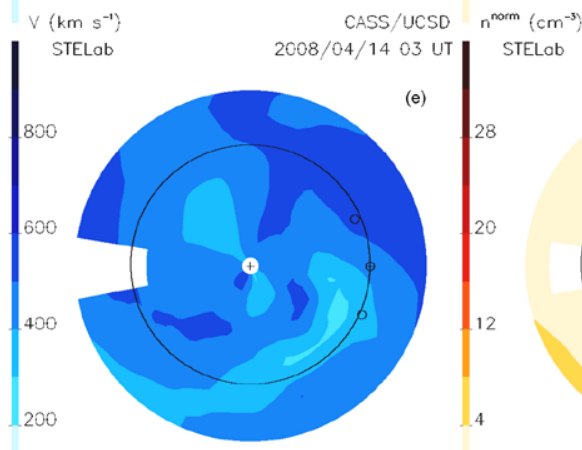

CASS/UCSD 2008/04/14 03 UT
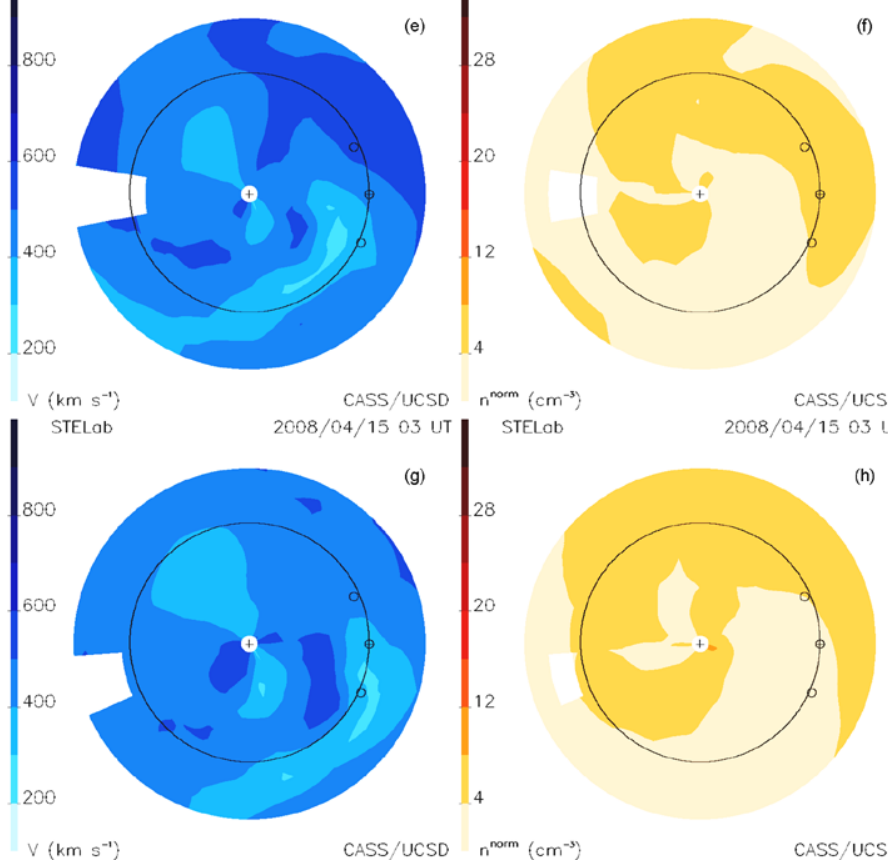

(f)

(d)

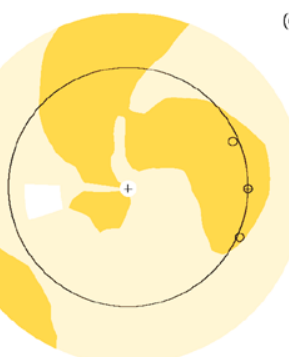
) 

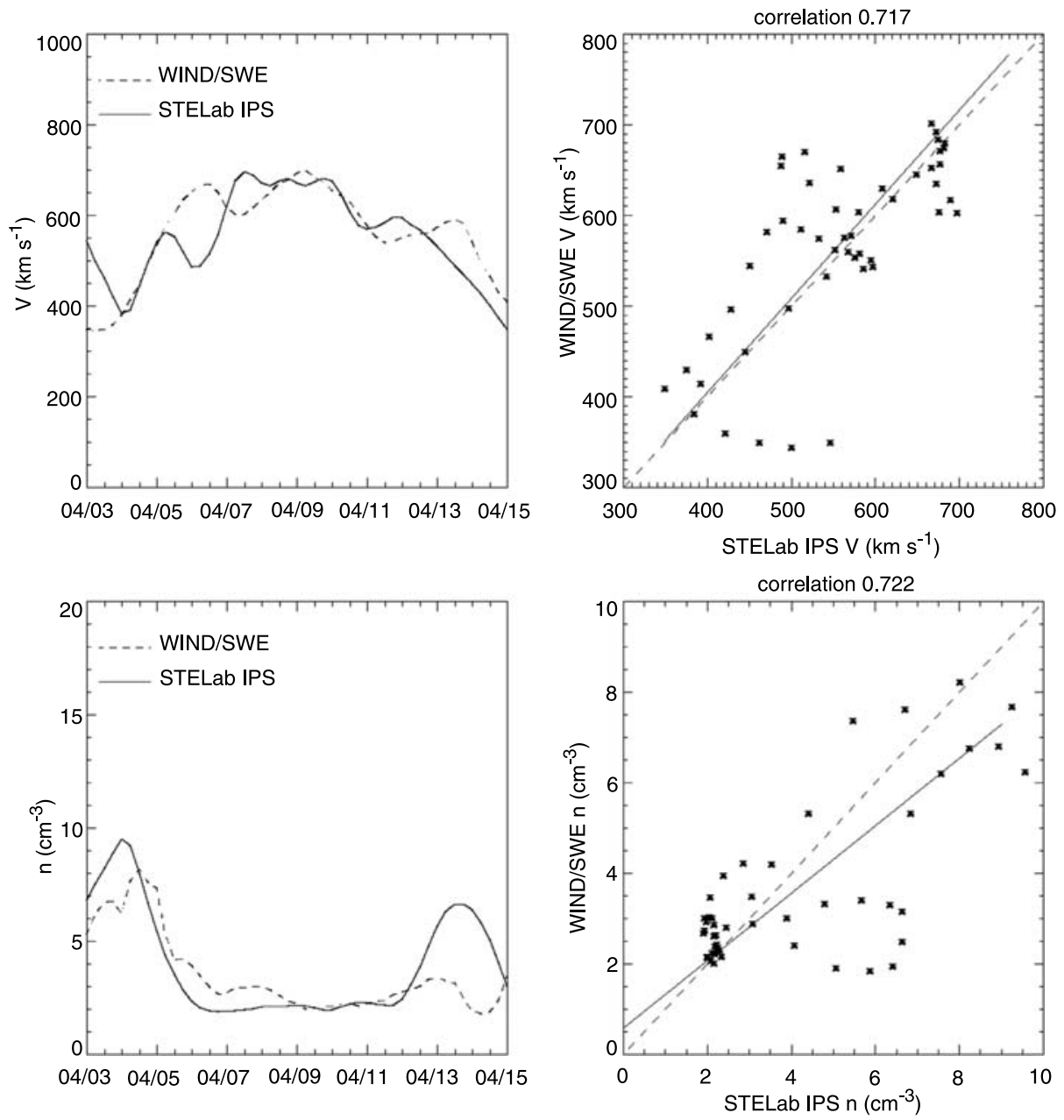

Figure 8 The left plots compare 3D reconstructed solar wind velocity (top) and density (bottom) at the Wind spacecraft (solid line), and those measured by Wind/SWE (dashed line). Wind/SWE data are originally hourly-averaged data that have been further averaged over a period of a day to match that of the IPS reconstruction. On the right are plots of the correlation of the two data sets; for each case the dashed lines represent a $100 \%$ correlation and the solid lines represent the best fit of the correlation.

and extracted from the tomography at its location does not have a positive correlation. We do not know exactly why this is, given that at both other spacecraft locations (Wind and STEREO-B) the density compares well, but clearly the density peak does not appear to be manifest from STEREO-B to Wind to STEREO-A as one might expect for a co-rotating dense structure.

Simunac et al. (2008) note that there are some CIRs during this interval where the estimated time of arrival from one STEREO spacecraft to the other (under the assumption of radial flow) is not consistent with actual arrival times. This discrepancy could result from potential changes in the solar wind speed/make-up or motion (or changes) of the coronal hole on the solar surface responsible for generating the CIR feature. 

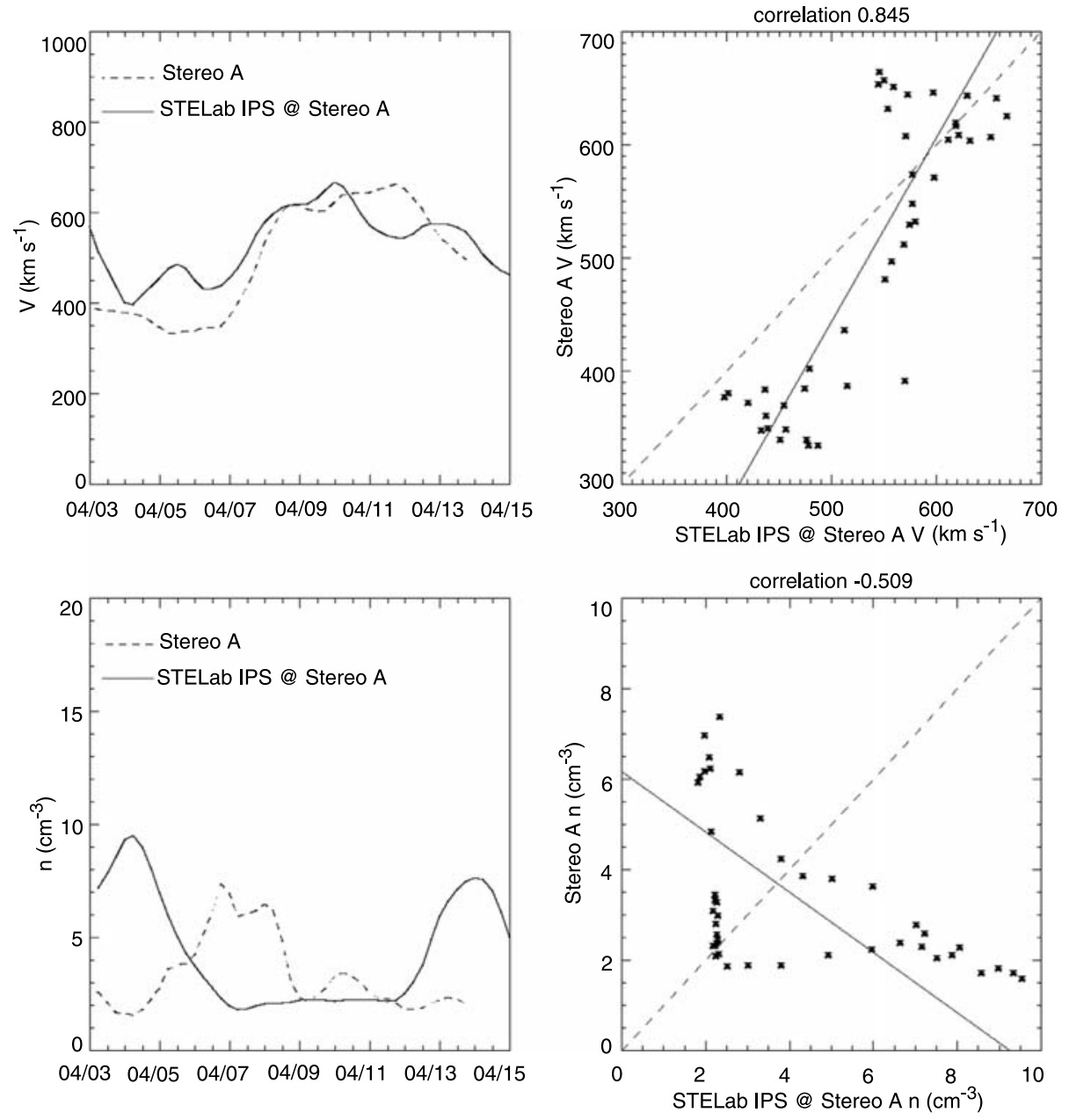

Figure 9 Plots as in Figure 8 but for STEREO-A/PLASTIC and 3D reconstruction at its location.

\section{Discussion and Summary}

WHI is a time of relatively quiet solar activity toward the end of solar cycle 23 and the start of solar cycle 24. Several weak CMEs were seen by LASCO (reported on the CDAW CME catalogue) and at least four CIRs were reported in more-detailed analyses of STEREO/PLASTIC in situ data by Simunac et al. (2008).

The low-resolution 3D-reconstructed heliosphere with STELab IPS velocity data, from a central part of the WHI period (around 4 April 2008), shows what appears to be a corotating region passing across the Sun-Earth $L_{1}$ point (and crossing STEREO-B first and STEREO-A later).

Some transient-type structure is observed later in this interval and this possibly interacts with these co-rotating features; this and the apparent non-co-rotation of density at the beginning warrants further investigation when higher-resolution tomography using data from Ooty or SMEI become available (see later). We expect that the 3D digital resolution of 

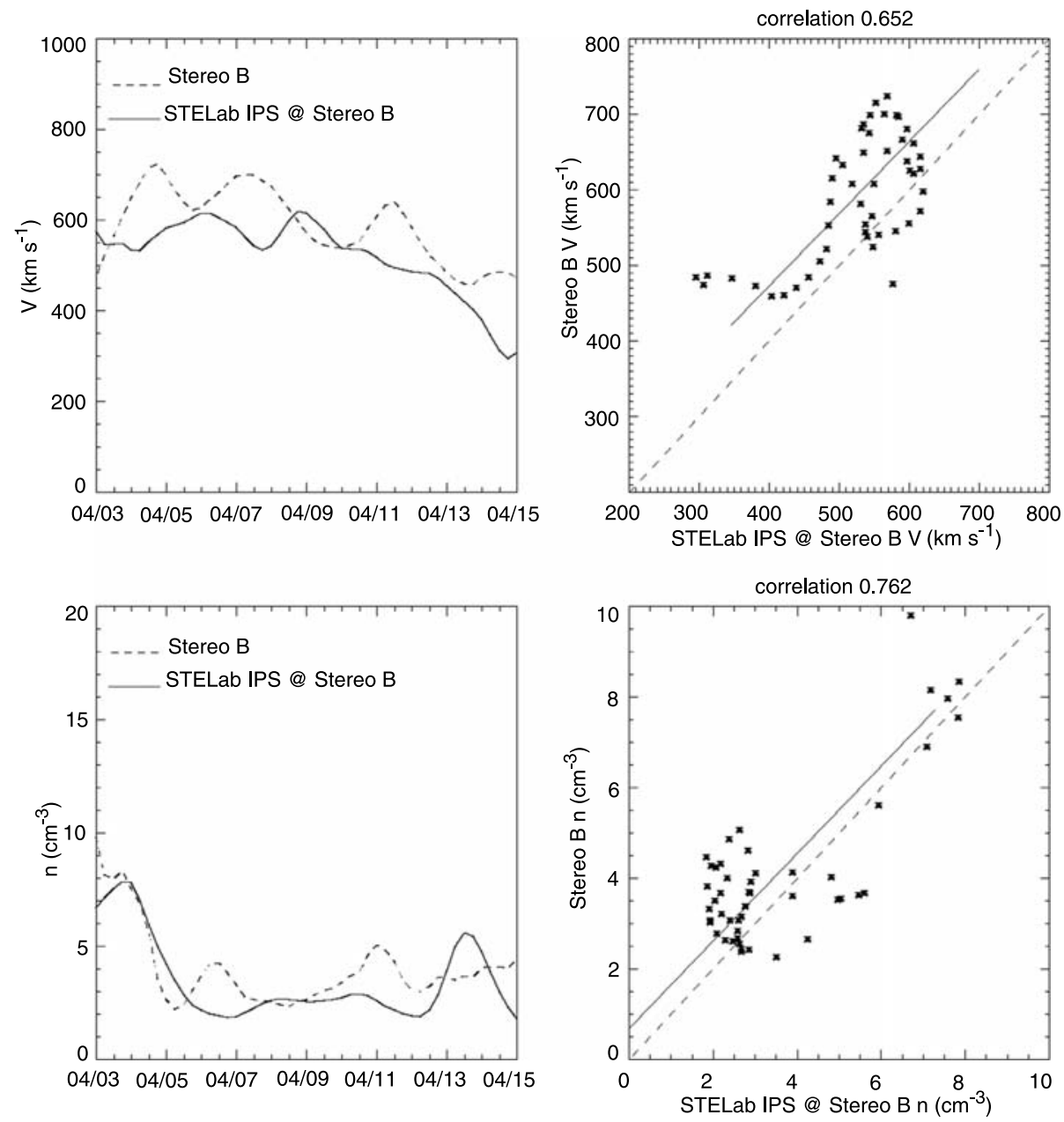

Figure 10 Plots as in Figure 9 but for STEREO-B/PLASTIC and 3D reconstruction at its location.

$20^{\circ} \times 20^{\circ}$ latitude - longitude centres with a one-day time cadence does not always adequately represent all the solar wind changes (especially in longitude) present during WHI at individual in situ locations and may be the reason for the STEREO-A density discrepancies. In particular, it is relatively clear that the coverage of the heliosphere by different lines of sight plays an important role in its reconstruction and to accurately reproduce the velocity/density profiles near the Earth (or at other locations in the inner heliosphere) as observed by the various spacecraft.

Time-series extraction from the reconstruction of radial velocity and density compare well for STEREO and Wind data, but not well for the aforementioned density discrepancies with STEREO-A/PLASTIC density. It should also be noted that STEREO-A/PLASTIC suffered a data outage beginning late on 13 April 2008 that lasted to the end of the WHI time interval.

Even so, good 3D reconstructions at three locations in the ecliptic have been achieved in velocity and two in density (the not-so-good comparison in density with STEREO-A to the 
west is likely due to the insufficient $g$-level observation coverage to the west of the SunEarth line early in the observing interval as detailed in Section 3). The resulting comparisons with STEREO-A, Wind, and STEREO-B are overall reasonable. Five out of the six sets of time-series comparisons correlate well and only the one density comparison with STEREOA does not. This suggests that the 3D reconstructions work well both at the Earth and moving away from it along its orbit as the STEREO in situ measurements become more distant from Earth. The STEREO spacecraft continue to move away from Earth at approximately $22^{\circ}$ per year which will lead to further opportunities to evaluate the performance of these $3 \mathrm{D}$ reconstructions in the ecliptic with a greater spacecraft spacing and a future set of improved spatial and temporal coverage from STELab IPS observations.

These successful comparisons of the 3D reconstructions with multi-point in-ecliptic in situ measurements add to the previous successful comparisons at Earth, Mars, and with the Ulysses spacecraft. Hence, the tomography used here is increasingly shown to be a useful and powerful tool for investigating solar wind structure in the inner heliosphere.

Further details on other IPS analyses of WHI (and increased-resolution tomography) such as those using Ooty IPS data will be discussed in a future article (in preparation). Studies are also planned using IPS observations from the European Incoherent SCATter radar (EISCAT) during WHI and white light from SMEI.

Acknowledgements The University of California, San Diego (UCSD) authors (M.M.B., B.V.J., A.B., J.M.C., and P.P.H.) acknowledge AFOSR grant FA9550-06-1-0107, NASA grants NNX08AJ116 and NNG05GM58G, and NSF grant ATM0331513 for supporting this work. The IPS observations were carried out under the solar wind program of STELab, Nagoya University, Japan. The authors also wish to thank the Wind/SWE group for the solar wind proton density and velocity measurements, and the STEREO/PLASTIC Consortium for making their data available via the World Wide Web. We also acknowledge use of the CME catalogue which is generated and maintained at the Coordinated Data Analysis Workshop (CDAW) Data Center by NASA and The Catholic University of America in cooperation with the Naval Research Laboratory. SOHO is a project of international cooperation between ESA and NASA.

Open Access This article is distributed under the terms of the Creative Commons Attribution Noncommercial License which permits any noncommercial use, distribution, and reproduction in any medium, provided the original author(s) and source are credited.

\section{References}

Armstrong, J.W., Coles, W.A.: 1972, Analysis of three-station interplanetary scintillation data. J. Geophys. Res. 77, 4602 .

Bisi, M.M., Breen, A.R., Habbal, S.R., Fallows, R.A.: 2004, Large-scale structure of the fast solar wind during the last solar minimum from EISCAT IPS measurements. In: “Auld Reekie” MIST/UKSP Joint Meeting, Edinburgh, Scotland (oral presentation).

Bisi, M.M., Breen, A.R., Fallows, R.A., Dorrian, G.D., Jones, R.A., Wannberg, G., Thomasson, P., Jordan, C.: 2006, Off-radial flow of the solar wind from EISCAT and MERLIN IPS observations. EOS Trans. AGU 87(52), Fall Meeting Suppl., Abstract SH33A-0399.

Bisi, M.M., Fallows, R.A., Breen, A.R., Habbal, S.R., Jones, R.A.: 2007a, Large-scale structure of the fast solar wind. J. Geophys. Res. 112, A06101. doi:10.1029/2006JA012166.

Bisi, M.M., Jackson, B.V., Fallows, R.A., Breen, A.R., Hick, P.P., Wannberg, G., Thomasson, P., Jordan, C.A., Dorrian, G.D.: 2007b, Combined STELab, EISCAT, ESR, and MERLIN IPS observations of the solar wind. Proc. SPIE 6689, 668911-1. doi:10.1117/12.735443.

Bisi, M.M., Jackson, B.V., Hick, P.P., Buffington, A., Odstrcil, D., Clover, J.M.: 2008, Three-dimensional reconstructions of the early November 2004 Coordinated Data Analysis Workshop geomagnetic storms: Analyses of STELab IPS speed and SMEI density data. J. Geophys. Res. 113, A00A11. doi:10.1029/2008JA013222.

Bisi, M.M., Jackson, B.V., Hick, P.P., Buffington, A., Clover, J.M.: 2009a, Coronal mass ejection reconstructions from interplanetary scintillation data using a kinematic model: A brief review. In: Proceedings of the AOGS 2007 Meeting, Ad. Geo., in press. 
Bisi, M.M., Jackson, B.V., Fallows, R.A., Dorrian, G.D., Manoharan, P.K., Clover, J.M., Hick, P.P., Buffington, A., Breen, A.R., Tokumaru, M.: 2009b, Solar wind and CME studies of the inner heliosphere using IPS data from STELab, ORT, and EISCAT. In: Proceedings of the AOGS 2008 Meeting, Ad. Geo., in press.

Breen, A.R., Canals, A., Fallows, R.A., Moran, P.J., Kojima, M.: 2002, Large-scale structure of the solar wind from interplanetary scintillation measurements during the rising phase of cycle 23. Adv. Space Res. 29, 379.

Breen, A.R., Fallows, R.A., Bisi, M.M., Thomasson, P., Jordan, C.A., Wannberg, G., Jones, R.A.: 2006, Extremely long baseline interplanetary scintillation measurements of solar wind velocity. J. Geophys. Res. 111, A08104. doi:10.1029/2005JA011485.

Coles, W.A.: 1996, A bimodal model of the solar wind speed. Astrophys. Space Sci. 243(1), 87.

Coles, W.A., Maagoe, S.: 1972, Solar wind velocity from ips observations. J. Geophys. Res. 77, 5622.

Coles, W.A., Rickett, B.J.: 1976, IPS observations of the solar wind speed out of the ecliptic. J. Geophys. Res. 81, 4797.

Crider, D.H., Vignes, D., Krymskii, A.M., Breus, T.K., Ness, N.F., Mitchell, D.L., Slavin, J.A., Acuña M.H.: 2003, A proxy for determining solar wind dynamic pressure at Mars using Mars global surveyor data. J. Geophys. Res. 108, 1461. doi:10.1029/2003JA009875.

Fallows, R.A., Breen, A.R., Dorrian, G.D.: 2008, Developments in the Use of EISCAT for interplanetary scintillation. Ann. Geophys. 26, 2229.

Galvin, A.B., Kistler, L.M., Popecki, M.A., Farrugia, C.J., Simunac, K.D.C., Ellis, L., Möbius, E., Lee, M.A., Boehm, M., Carroll, J., Crawshaw, A., Conti, M., Demaine, P., Ellis, S., Gaidos, J.A., Googins, J., Granoff, M., Gustafson, A., Heirtzler, D., King, B., Knauss, U., Levasseur, J., Longworth, S., Singer, K., Turco, S., Vachon, P., Vosbury, M., Widholm, M., Blush, L.M., Karrer, R., Bochsler, P., Daoudi, H., Etter, A., Fischer, J., Jost, J., Opitz, A., Sigrist, M., Wurz, P., Klecker, B., Ertl, M., Seidenschwang, E., Wimmer-Schweingruber, R.F., Koeten, M., Thompson, B., Steinfeld, D.: 2008, The plasma and suprathermal ion composition (PLASTIC) investigation on the STEREO observatories. Space Sci. Rev. 136, 437. doi:10.1007/s11214-007-9296-x.

Hewish, A., Scott, P.F., Wills, D.: 1964, Interplanetary scintillations of small diameter radio sources. Nature 203, 1214.

Hick, P.P., Jackson, B.V.: 2004, Heliospheric tomography: An algorithm for the reconstruction of the 3D solar wind from remote sensing observations. In: Fineschi, S., Gummin, M.A. (eds.) Telescopes and Instrumentation for Solar Astrophysics, Proc. SPIE 5171, 287. doi:10.1117/12.513122.

Jackson, B.V., Hick, P.P.: 2005, Three-dimensional tomography of interplanetary disturbances. In: Gary, D., Keller, C.U. (eds.) Solar and Space Weather Radiophysics: Current Status and Future Developments, Astrophys. and Space Sci. Lib. 314, Kluwer Academic Publishers, Dordrecht, 355. Chap. 17.

Jackson, B.V., Hick, P.P., Kojima, M., Yokobe, A.: 1998, Heliospheric tomography using interplanetary scintillation observations, 1. Combined Nagoya and Cambridge data. J. Geophys. Res. 103(A6), 12049.

Jackson, B.V., Hick, P.P., Buffington, A., Kojima, M., Tokumaru, M., Fujiki, K., Ohmi, T., Yamashita, M.: 2003, Time-dependent tomography of hemispheric features using interplanetary scintillation (IPS) remote-sensing observations. In: Velli, M., Bruno, R., Malara, F., Bucci, B. (eds.) Solar Wind Ten, AIP Conf. Ser. 679, 75. doi:10.1063/1.1618545.

Jackson, B.V., Buffington, A., Hick, P.P., Wang, X., Webb, D.: 2006, Preliminary three-dimensional analysis of the heliospheric response to the 28 October 2003 CME using SMEI white-light observations. J. Geophys. Res. 111, A04S91. doi:10.1029/2004JA010942.

Jackson, B.V., Boyer, J.A., Hick, P.P., Buffington, A., Bisi, M.M., Crider, D.H.: 2007, Analysis of solar wind events using interplanetary scintillation (IPS) remote sensing 3D reconstructions and their comparison at Mars. Solar Phys. 241(2), 385. doi:10.1007/s11207-007-0276-9.

Jackson, B.V., Bisi, M.M., Hick, P.P., Buffington, A., Clover, J.M., Sun, W.: 2008, Solar mass ejection imager (SMEI) 3D reconstruction of the 27-28 May 2003 CME Sequence. J. Geophys. Res. 113, A00A15. doi:10.1029/2008JA013224.

Jones, R.A., Breen, A.R., Fallows, R.A., Canals, A., Bisi, M.M., Lawrence, G.: 2007, Interaction between coronal mass ejections and the solar wind. J. Geophys. Res. 112, A08107. doi:10.1029/2006JA011875.

Kaiser, M.L.: 2005, The STEREO mission: An overview. Adv. Space Res. 36, 1483. doi:10.1016/j.asr.2004. 12.066.

Kaiser, M.L., Kucera, T.A., Davila, J.M., St. Cyr, O.C., Guhathakurta, M., Christian, E.: 2008, The STEREO mission: An introduction. Space Sci. Rev. 136, 5. doi:10.1007/s11214-007-9277-0.

Klinglesmith, M.: 1997, The polar solar wind from 2.5 to 40 solar radii: Results of intensity scintillation measurements. Ph.D. Thesis, University of California, San Diego (UCSD).

Kojima, M., Kakinuma, T.: 1987, Solar cycle evolution of solar wind speed structure between 1973 and 1985 observed with the interplanetary scintillation method. J. Geophys. Res. 92, 7269. 
Kojima, M., Breen, A.R., Fujiki, K., Hayashi, K., Ohmi, T., Tokumaru, M.: 2004, Fast solar wind after the rapid acceleration. J. Geophys. Res. 109, A04103. doi:10.1029/2003JA010247.

Manoharan, P.K., Ananthakrishnan, S.: 1990, Determination of solar-wind velocities using single-station measurements of interplanetary scintillation. Mon. Not. Roy. Astron. Soc. 244, 691.

McComas, D.J., Bame, S.J., Barker, P., Feldman, W.C., Phillips, J.L., Riley, P., Griffee, J.W.: 1998, Solar wind electron proton alpha monitor (SWEPAM) for the advanced composition explorer. Space Sci. Rev. 86, 563. doi:10.1023/A:1005040232597.

Odstrcil, D., Pizzo, V.J.: 2002, Numerical simulation of interplanetary disturbances. In: Sawaya-Lacoste, H. (ed.) Solspa 2001, Proceedings of the Second Solar Cycle and Space Weather Euroconference, ESA SP 477, 281.

Ogilvie, K.W., Desch, M.D.: 1997, The wind spacecraft and its early scientific results. Adv. Space Res. 20, 559.

Ogilvie, K.W., Chornay, D.J., Fritzenreiter, R.J., Hunsaker, F., Keller, J., Lobell, J., Miller, G., Scudder, J.D., Sittler, E.C. Jr., Torbert, R.B., Bodet, D., Needell, G., Lazarus, A.J., Steinberg, J.T., Tappan, J.H., Mavretic, A., Gergin, E.: 1995, SWE, a comprehensive plasma instrument for the wind spacecraft. Space Sci. Rev. 71, 55.

Simunac, K.D., Galvin, A.B., Kistler, L.M., Popecki, M.A., Lee, M.A., Russell, C.T.: 2008, Do in situ observations of solar wind stream interfaces reflect the evolution of coronal holes? Eos Trans. AGU 89(53), Fall Meeting Suppl., Abstract SH23A-1618.

Stone, E.C., Frandsen, A.M., Mewaldt, R.A., Christian, E.R., Margolies, D., Ormes, J.F., Snow, F.: 1998, The advanced composition explorer. Space Sci. Rev. 86, 1. doi:10.1023/A:1005082526237.

Wenzel, F.P., Marsden, R.G., Page, D.E., Smith, E.J.: 1992, The ULYSSES mission. Astron. Astrophys. Suppl. 92, 207. 